\title{
Whole-Genomic Analysis of NDM-5-Producing Enterobacteriaceae Recovered from an Urban River in China
}

\author{
Ying $\mathrm{Li}^{1,2, *}$ \\ Min Tang ${ }^{3, *}$ \\ Xiaoyi Dai ${ }^{4,5}$ \\ Yingshun Zhou ${ }^{4,5}$ \\ Zhikun Zhang ${ }^{4,5}$ \\ Yichuan Qiu ${ }^{4,5}$ \\ Chengwen $\mathrm{Li}^{1,2}$ \\ Luhua Zhang ${ }^{4,5}$
}

'Department of Immunology, School of Basic Medical Sciences, Southwest Medical University, Luzhou, Sichuan, People's Republic of China;

${ }^{2}$ Immunological Technology Platform, Southwest Medical University, Luzhou, Sichuan, People's Republic of China; ${ }^{3}$ Department of Laboratory Medicine, the Affiliated Hospital of Southwest Medical University, Luzhou, Sichuan, People's Republic of China; ${ }^{4}$ Department of Pathogenic Biology, School of Basic Medical Sciences, Southwest Medical University, Luzhou, Sichuan, People's Republic of China; ${ }^{5}$ Pathogen Biology Platform, Southwest Medical University, Luzhou, Sichuan, People's Republic of China

*These authors contributed equally to this work
Purpose: Three NDM-5-producing Enterobacteriaceae (Escherichia coli, Klebsiella pneumoniae, and Citrobacter braakii, one each) were isolated during a screening study for the presence of carbapenemase-producing Enterobacteriaceae (CPE) strains in urban rivers in China. The aim of the present study was to characterize these NDM-5-producing isolates by using whole-genome analysis.

Methods: In vitro susceptibility testing was performed using the broth microdilution method. Conjugation assay was carried out to investigate the transferability of bla $a_{\mathrm{NDM}-5^{-}}$ harboring plasmids. Whole-genome sequencing was performed using an Illumina HiSeq combined with the PacBio RSII system. The genetic characteristics of the bla $a_{\mathrm{NDM}-5^{-}}$ harboring plasmids were analyzed. Antimicrobial resistance genes and virulence genes were identified from the genome sequences. Phylogenetic analysis was performed based on core genome.

Results: Antimicrobial susceptibility testing showed that all three isolates were resistant to carbapenems, cephalosporins, quinolones, and aminoglycosides, and susceptible to colistin. Whole-genome sequencing showed that each isolate carried multiple antibiotic resistance genes mediating multidrug resistance, and harbored numerous virulence genes, some of which were located on plasmids. In these isolates, $b l a_{\mathrm{NDM}-5}$ was carried by an IncX 3 plasmid in $K$. pneumoniae and C. braakii, and on an IncR/IncX1 plasmid in E. coli. Conjugation experiments showed that these $b l a_{\mathrm{NDM}-5}$-haboring plasmids were successfully transferred to E. coli J53. Phylogenetic analysis revealed that E. coli SCLZR49 was present in a cluster with isolates of different origin, K. pneumoniae SCLZR50 was mainly clustered with clinical isolates, and $C$. braakii SCLZR53 had closely genetic relationship with environmental isolates.

Conclusion: This study revealed contamination of the urban river ecosystems by clinically significant carbapenemase gene $b l a_{\mathrm{NDM}-5}$, raising the possibility of plasmid transmission into the environmental from humans and highlighting the need for a constant surveillance of CPE in the environment under the "One-Health" perspective.

Keywords: carbapenemase, antimicrobial resistance, aquatic environment, river water

\section{Introduction}

Carbapenems are considered as the mainstay agents of choice for treating serious infections caused by extended-spectrum- $\beta$-lactamase (ESBL)-producing Enterobacteriaceae. ${ }^{1}$ Therefore, the emergence and rapid global dissemination of carbapenemase-producing Enterobacteriaceae (CPE) represent a significant threat to public health system around the world. ${ }^{2}$ Carbapenemase-encoding genes are often

\author{
Correspondence: Luhua Zhang; \\ Chengwen $\mathrm{Li}$ \\ Xiang Lin Road, Longmatan District, \\ Luzhou City, Sichuan Province, 646000, \\ People's Republic of China \\ Tel/Fax +86-0830-3160073 \\ Email zhluhua@swmu.edu.cn; \\ Lichwl10119@I63.com
}


associated with various mobile genetic elements (MGEs), such as plasmids, insertion sequences, integrons and transposons, and are now spreading worldwide at an alarming rate. ${ }^{3}$ New Delhi metallo- $\beta$-lactamase (NDM), one of the most important carbapenemases, can hydrolyze nearly all classes of $\beta$-lactams (including carbapenems), with the exception of monobactams. ${ }^{4}$ To date, $b l a_{\mathrm{NDM}}$ genes have been extensively studied in clinical specimens and settings globally, ${ }^{4}$ however, the prevalence and genetic characteristics of $b l a_{\mathrm{NDM}}$ in the natural ecosystems have not been adequately investigated.

Clinical facilities are traditionally viewed as reservoirs of antibiotic resistance genes (ARGs). However, aquatic environments have been gradually recognized as a vital transport vessel for diverse ARGs. ${ }^{5}$ The increased human activities cause serious ARG burden to the environmental waters, which poses serious challenges to public health as they are commonly used for recreational, irrigation and drinking water purposes. In China, CPE isolates harboring $b l a_{\mathrm{KPC}-2}{ }^{6-8}$ and $b l a_{\mathrm{NDM}-1}{ }^{9-11}$ from water samples were widely reported, while $b l a_{\mathrm{NDM}-5}$-bearing isolates had not been fully explored. Rivers, especially when associated with effluent of hospital or urban wastewater, are remarkable hotspots for horizontal transfer of ARGs including carbapenemase genes. ${ }^{12,13}$ Previous reports have identified several carbapenemase genes in Enterobacteriaceae isolates from river systems in many countries in recent years, including $b l a_{\mathrm{KPC}-2}, b l a_{\mathrm{NDM}-1}$ and $b l a_{\mathrm{NDM}-5}$ in China, ${ }^{8,10,14}$ bla $_{\mathrm{KPC}-2}$, bla $_{\mathrm{NDM}-[1,5,7]}$, bla $_{\mathrm{OXA}-[48,181]}$, bla $_{\mathrm{GES}-20}$, and $b l a_{\mathrm{IMI}-18}$ in Philippines, ${ }^{15}$ $b l a_{\mathrm{KPC}-3}, \quad b l a_{\mathrm{NDM}-1}$, and $b l a_{\mathrm{GES}-5}$ in Portuguese, ${ }^{16}$ bla $_{\mathrm{KPC}-[2,3]}, b l a_{\mathrm{NDM}-5}, b a_{\mathrm{OXA}-[48,181]}$, and bla $_{\mathrm{VIM}-1}$ in Switzerland, ${ }^{12}$ bla $_{\mathrm{KPC}-2}$, bla $_{\mathrm{IMI}-2}$, and bla $a_{\mathrm{VIM}-1}$ in Spain, ${ }^{17}$ $b l a_{\text {NDM-5 }}$ in France, ${ }^{18}$ bla $_{\text {NDM-9 }}$ in South Korea ${ }^{19}$ and bla $_{\text {OXA-48 }}$ in Algeria. ${ }^{20}$ The $b l a_{\mathrm{NDM}-5}$-bearing Enterobacteriaceae isolates from river water in China requires investigation.

Compared with clinical isolates, data are limited for CPE in environmental waters with respect to detailed genetic characteristics of ARGs-harboring MGEs and virulence gene profiles. During a screening study for the presence of CPE strains in urban river water, we isolated three $b l a_{\mathrm{NDM}-5}$-carrying Enterobacteriaceae strains from two surface water samples from Tuojiang River in Luzhou city, Sichuan Province, China, in August 2019. ${ }^{21}$ The aim of the present study was to characterize these three NDM-5-producing Enterobacteriaceae isolates by using whole-genome sequencing and analysis. We provided genetic information about resistance determinants and virulence gene profiles, as well as phylogenetic relationships of NDM-5-producing isolates. We determined the genomic environment of $b l a_{\mathrm{NDM}-5}$-carrying plasmids and also revealed the genetic characterization of resistance plasmids co-existing in the CPE strains. This work will help to make a better understanding of the mechanisms of the spread of bla NDM-5 among bacterial community in aquatic environments.

\section{Materials and Methods}

\section{Sample Collection and Bacterial Isolation}

During a screening study for the presence of CPE strains in hospital sewage and receiving rivers, we isolated three $b l a_{\mathrm{NDM}-5}$-carrying Enterobacteriaceae strains from two surface water samples from Tuojiang River in Luzhou city, Sichuan Province, China, in August 2019. ${ }^{21}$ The Tuojiang River runs through the Luzhou City, where it flows into the Yangtze River. The two sampling sites are at least $1 \mathrm{~km}$ apart and there is a hospital effluent outlet between them. The hospital is a 3000-beds tertiary teaching hospital and serves as the major referral medical center in Luzhou City. Per sampling site, $500 \mathrm{~mL}$ river water was taken in sterile glass bottles and brought to the laboratory for subsequent analysis within the following $1 \mathrm{~h} .200 \mathrm{~mL}$ water sample was centrifuged at $5000 \mathrm{~g}$ for $5 \mathrm{~min}$ and the precipitation was resuspended in sterile $100 \mu \mathrm{L}$ $0.9 \% \mathrm{NaCl}$ solution. The enrichments were plated onto MacConkey agar containing meropenem (2 $\mu \mathrm{g}$ / $\mathrm{mL}$ ) and cultured for $24 \mathrm{~h}$ at $37{ }^{\circ} \mathrm{C}$. Pink colonies with various morphologies were picked and repeatedly streaked on new MacConkey agar plates to obtain pure isolates.

\section{Characterization of CPE and}

\section{Antimicrobial Susceptibility Testing}

As described in our previous study, ${ }^{21}$ initial species identification was performed by PCR amplification of $16 \mathrm{~S}$ rRNA gene and Sanger sequencing, ${ }^{22}$ and the presence of the acquired carbapenemase genes, bla $a_{\mathrm{KPC}}, b l a_{\mathrm{NDM}}$, $b l a_{\mathrm{OXA}-48}, b l a_{\mathrm{OXA}-58}, b l a_{\mathrm{VIM}}$, and $b l a_{\mathrm{IMP}}$ was screened via PCR assays. ${ }^{23}$ Eighteen $b^{2} a_{\mathrm{NDM}-5}$-bearing strains were isolated, including nine Escherichia coli (eight from hospital sewage and one from river), one Cronobacter sakazakii (from hospital sewage), four Klebsiella pneumoniae (three from hospital sewage and one from river), one 
Klebsiella variicola (from hospital sewage), one Pseudomonas sp. (from hospital sewage), one Acinetobacter sp. (from hospital sewage) and one Citrobacter freundii (from river, later confirmed as a Citrobacter braakii). These three bla $_{\mathrm{NDM}-5}$-positive isolates (E. coli, K. pneumoniae and C. braakii, one each) recovered from river were chosen for further genome analysis. Antimicrobial susceptibility profiles were generated by performing in vitro susceptibility testing against 11 antimicrobials using the microdilution broth method following recommendations of the Clinical Laboratory Standards Institute (CLSI). ${ }^{24}$ The breakpoints of colistin and tigecycline were interpreted according to the European Committee on Antimicrobial Susceptibility Testing (EUCAST). ${ }^{25}$ E. coli ATCC 25922 was used as a quality control for minimum inhibitory concentrations (MICs) determination.

\section{Whole-Genome Sequencing (WGS) and Data Analyses}

To characterize the genetic features of $b l a_{\mathrm{NDM}-5^{-}}$ carrying Enterobacteriaceae isolates, genomic DNA was extracted using the QIAamp DNA Mini Kit (Qiagen). Purified DNA was subjected to wholegenome sequencing using a sheared DNA library with an average size of $10 \mathrm{~kb}$ on a PacBio RSII sequencer (Pacific Biosciences, Menlo Park, USA), as well as with a paired-end library with an insert size of 150 bp on a HiSeq 2000 sequencer (Illumina, San Diego, CA, USA). The de novo assembly of the long PacBio reads was performed using the single-molecule realtime (SMRT) Link v5.0.1. Illumina reads were mapped over the PacBio-generated contigs to correct the assembled contigs using bwa. Library construction and sequencing was performed at Beijing Novogene Bioinformatics Technology Co. Ltd.

Assembled genome sequences were annotated by using the Prokka ${ }^{26}$ combined with BLASTP searches against the UniProtKB/Swiss-Prot database. Sequence type (ST), ARGs and plasmid incompatibility types were determined using the Center for Genomic Epidemiology (http://www. genomicepidemiology.org/) web tools MLST (Multi-Locus Sequence Typing), ResFinder, PlasmidFinder and pMLST, respectively. Insertion elements (IS) were annotated using online databases IS Finder. ${ }^{27}$ The presence of virulence genes was investigated by using VirulenceFinder (https:// cge.cbs.dtu.dk/services/VirulenceFinder/) and the virulence factor database (VFDB, http:/www.mgc.ac.cn/ VFs/main.htm). Average nucleotide identity (ANI) analysis was calculated with JSpeciesWS (http://jspecies.ribo host.com/jspeciesws/\#analyse). Digital DNA-DNA hybridization $(\mathrm{dDDH})$ values were calculated using GGDC 3.0 server (http://ggdc.dsmz.de/distcalc2.php) by means of genome-to-genome sequence comparison. Multiple and pairwise sequence comparisons were performed using the BRIG tool. ${ }^{28}$ Gene organization diagrams were visualized with Inkscape 0.92.4 (https://inkscape.org/en/).

\section{Conjugation Assays}

Conjugation experiments were performed using filter- and broth-based methods with the azide-resistant $E$. coli strain J53 as the recipient. Both the donors and J53 were grown to exponential stage (the optical density at $600 \mathrm{~nm}$ reaches $\sim 0.5$ ) and then mixed at a donor/recipient ratio of $1: 1$. After incubation at $37^{\circ} \mathrm{C}$ for $24 \mathrm{~h}$, transconjugants were selected using $2 \mu \mathrm{g} / \mathrm{mL}$ meropenem plus $150 \mu \mathrm{g} / \mathrm{mL}$ sodium azide. The presence of $b l a_{\mathrm{NDM}-5}$ in transconjugants was confirmed by PCR using the primers bla $a_{\mathrm{NDM}}-\mathrm{F} 5^{\prime}$ ATTTACTAGGCCTCGCATTTGC-3' and bla NDM $^{\prime} \mathrm{R}$ 5'GCCTCTGTCACATCGAAATCG-3'. The MICs of 11 antimicrobials against the $E$. coli J53 transconjugants were determined using the microdilution broth method as described above.

\section{Phylogenetic Analysis}

Genomes were annotated using Prokka and annotated GFF3 files were piped into Roary to create a core genome alignment. A maximum-likelihood phylogenetic tree based on the core genome alignment was constructed using RaxML under the GTRGAMMA model with 1000 bootstrap iterations. The presence of $\beta$-lactamase genes was determined by Abricate. Carriage of $\beta$-lactamase genes and detail information of isolates were annotated on the trees using iTOL (https://itol. embl.de/). Single nucleotide polymorphisms (SNPs) were extracted using snp-sites v2.3.2. ${ }^{29}$

\section{Nucleotide Sequence Accession Numbers}

The genome sequence data of E. coli SCLZR49, $K$. pneumoniae SCLZR50 and C. braakii SCLZR53 in this study have been deposited in the National Microbiology Data Center under the accession numbers NMDC60018294, NMDC60018295 and NMDC60018296, respectively. 


\section{Results}

\section{Detection of NDM-5-Producing Bacteria from River Water}

bla $a_{\mathrm{NDM}-5}$-positive Enterobacteriaceae isolates, E. coli SCLZR49, $K$. pneumoniae SCLZR50, and $C$. freundii SCLZR53 (later confirmed as a $C$. braakii), were all recovered from Tuojiang river. Among them, SCLZR49 and SCLZR50 isolates were from the same upstream water sample $\left(\mathrm{E} 105^{\circ} 43^{\prime}, \mathrm{N} 28^{\circ} 88^{\prime}\right)$, and the SCLZR53 isolate was from the downstream $\left(\mathrm{E} 105^{\circ} 45^{\prime}, \mathrm{N} 28^{\circ} 90^{\prime}\right)$. These three NDM-5-producing isolates exhibited resistance to amikacin, gentamicin, meropenem, imipenem, cefoxitin, aztreonam, ciprofloxacin, and cefotaxime, but were susceptible to colistin (Table 1). In addition, the SCLZR50 strain was resistant to fosfomycin and tigecycline, which SCLZR49 and SCLZR53 were susceptible to.

\section{WGS Analysis of NDM-5-Producing Isolates}

High-throughput sequencing of the E. coli SCLZR49 revealed a chromosome of 4,883,356 bp and 4 circularly closed plasmids ranging in size from 3885 bp to 210,840 bp, encoding 4 to 238 predicted open reading frames (ORFs) (Table 2). In consistence with its multidrug resistance phenotype, SCLZR49 had 34 genes mediating resistance to $\beta$-lactams ( $b l a_{\mathrm{NDM}-5}, b l a_{\mathrm{OXA}-10}$, and $\left.b l a_{\mathrm{TEM}-1 \mathrm{~B}}\right)$, aminoglycosides [aac(3)-IV, aadA1, aadA2, aadA22, aph (3')-Ib, aph(3')-IIa, aph(3')-Ia, aph(4)-Ia, aph(6)-Id, and $r m t B]$, macrolides $[m d f(A), \ln u(F)$, and $\operatorname{mph}(A)]$, phenicols (cmlAl and floR), quinolones (oqxA, oqxB, and qnrS1), rifampicin (ARR-2), sulphonamides (sull and sul2), tetracyclines $[\operatorname{tet}(A)]$, and trimethoprim (dfrA12 and $d f r A 14)$ (Table 2). These ARGs are generally distributed on different plasmids, except the chromosomally located $m d f(A)$ gene.

The isolate SCLZR50 contained a chromosome of $5,089,532 \mathrm{bp}$ and 4 circularly closed plasmids of 53,134 to $248,272 \mathrm{bp}$ in length, containing 67 to 275 predicated ORFs (Table 2). In silico analysis identified a total of 41 ARGs (Table 2), conferring resistance to $\beta$-lactams $\left(b l a_{\mathrm{SHV}-26}, b l a_{\mathrm{CTX}-\mathrm{M}-3}, b l a_{\mathrm{DHA}-1}, b l a_{\mathrm{NDM}-5}, b l a_{\mathrm{OXA}-10}\right.$, and $\left.b l a_{\mathrm{TEM}-1 \mathrm{~B}}\right)$, aminoglycosides [aac(3)-IV, $\operatorname{aac}\left(6^{\prime}\right)-I b-c r$, aadA1, aadA16, aadA2b, aph(3')-Ib, aph(3')-Ia, aph(4)$I a$, aph(6)-Id, armA, and $r m t B]$, fosfomycin (fos $A 3$ and fosA5), macrolides $[m p h(A), \operatorname{mph}(E)$, and $\operatorname{msr}(E)]$, phenicols (cmlA1 and floR), quinolones [aac(6')-Ib-cr, qnrB2, $q n r B 4, q n r B 52, q n r S 1, o q x A$, and oqxB], rifampicin (ARR-
3), sulphonamides (sull and sul3), tetracyclines [tet(A)], and trimethoprim (dfrA27). Among these ARGs, $b l a_{\mathrm{SHV}-26}$, fos $A 5$, oqxA, and oqxB were located on the chromosome.

Whole-genome sequencing revealed that the genome DNA of SCLZR53 comprises a 5,263,019-bp chromosome, three plasmids and three contigs. Due to possible complex structures or high numbers of transposases and ISs, these three contigs were not successfully assembled into circular forms. The plasmids and contigs in this isolate vary in size from $52 \mathrm{~kb}$ to $\sim 286 \mathrm{~kb}$, encoding 68 to $\sim 329$ predicted ORFs (Table 2). ANI analysis revealed that the strain SCLZR53 actually belongs to C. braakii, as it only had $90.31 \%$ identity (77.12\% query coverage) to $C$. freundii CFNIH1, but $98.35 \%$ identity $(84.52 \%$ query coverage) to $C$. braakii FDAARGOS_290, above the 96\% cut-off for defining a bacterial species. ${ }^{30}$ Consistent with ANI data, the dDDH value between SCLZR53 and FDAARGOS_290 was $90.80 \%$, above the suggested $70 \%$ cut-off for species delineation as well as the $79 \%$ cut-off for subspecies delineation. Resfinder analysis showed that SCLZR53 carried 31 ARGs encoding resistance to $\beta$-lactams $\left(b_{\mathrm{CMY}-101}\right.$, bla $_{\mathrm{NDM}-5}$, and $\left.b l a_{\mathrm{TEM}-1 \mathrm{~B}}\right)$, aminoglycosides [aac(3)-IIa, $\operatorname{aac}(3)-I V$, $\operatorname{aac}\left(6^{\prime}\right)-$ Ib-cr, aadA22, aadA5, aph(3")-Ib, aph(3')-Ia, aph(4)-Ia, aph (6)-Id, and $r m t B]$, fosfomycin (fos $A 3)$, macrolides [mph $(A)$ and $\operatorname{lnu}(F)]$, phenicols $(f l o R)$, quinolones $\left[a a c\left(6^{\prime}\right)-I b-c r\right.$, oqx $A$, and oqxB], rifampicin (ARR-3), sulphonamides (sul1), tetracyclines [tet(A)], and trimethoprim (dfrA17 and dfrA27). All the ARGs were carried by plasmids, except the $\beta$-lactam gene $b l a_{\mathrm{CMY}-101}$.

\section{Virulence Gene Profiles of NDM-5-Producing Isolates}

Sixty genes with attributes of virulence were detected in E. coli SCLZR49 (Table S1), including the fimBCDEFGHI operon encoding type 1 fimbriae, the entBCDEFS operon and the fepACG operon encoding enterobactin siderophore, the tssCFM operon encoding type VI secretion system (T6SS), the esp (LXR) encoding type III secretion system, the gspCDEFGHJKLM operon encoding general secretion pathway, as well as genes involved in the formation of Vi polysaccharide (tviCDE and vexABCD), and genes encoding EAST-1 heat-stable toxin (astA) and hemolysin $\mathrm{F}(h l y F)$. Based on its virulence gene profiles, SCLZR49 is not predicted to be an extraintestinal pathogenic $E$. coli (ExPEC) strain (defined as presence of two or more of the following markers: papA and/or papC, afa/ 
Table I MICs for SCLZR49, SCLZR50, SCLZR53, Their Transformants, and the Recipient Strain J53

\begin{tabular}{|c|c|c|c|c|c|c|c|c|c|c|c|c|}
\hline \multirow[t]{2}{*}{ Strains } & \multirow[t]{2}{*}{ Species' Name } & \multicolumn{11}{|c|}{$\operatorname{MIC}(\mu \mathrm{g} / \mathrm{mL})^{a}$} \\
\hline & & AMK & FOS & GEN & CST & MEM & IMP & CFT & AZT & CIP & CTX & TGC \\
\hline SCLZR49 & E. coli & $>512$ & 32 & $>512$ & $\leq 0.5$ & 512 & 512 & $>512$ & 512 & 512 & $>512$ & $\leq 0.5$ \\
\hline J53/pNDM5_SCLZR49 & E. coli & $>512$ & 128 & 512 & $\leq 0.5$ & 512 & 128 & $>512$ & 2 & $\leq 0.5$ & 512 & $\leq 0.5$ \\
\hline SCLZR50 & K. pneumoniae & $>512$ & $>512$ & $>512$ & $\leq 0.5$ & $>512$ & 512 & $>512$ & 256 & 256 & $>512$ & 4 \\
\hline J53 /pNDM5_SCLZR50 & E. coli & $>512$ & 128 & 512 & $\leq 0.5$ & 512 & 512 & $>512$ & 2 & $\leq 0.5$ & 512 & $\leq 0.5$ \\
\hline SCLZR53 & C. braakii & $>512$ & 128 & $>512$ & $\leq 0.5$ & 512 & 128 & $>512$ & 16 & 256 & 512 & 1 \\
\hline J53/pNDM5_SCLZR53 & E. coli & $>512$ & 128 & $>512$ & $\leq 0.5$ & 512 & 128 & 512 & 8 & $\leq 0.5$ & 512 & $\leq 0.5$ \\
\hline $\mathrm{J} 53$ & E. coli & 8 & 8 & 8 & $\leq 0.5$ & $\leq 0.5$ & $\leq 0.5$ & 2 & 8 & $\leq 0.5$ & $\leq 0.5$ & $\leq 0.5$ \\
\hline ATCC25922 & E. coli & 4 & 2 & 2 & 2 & $\leq 0.5$ & $\leq 0.5$ & 8 & 8 & $\leq 0.5$ & $\leq 0.5$ & $\leq 0.5$ \\
\hline
\end{tabular}

Note: Resistant MIC's are highlighted in bold.

Abbreviations: ${ }^{a}$ AMK, amikacin; FOS, fosfomycin; GEN, gentamicin; CST, colistin; MEM, meropenem; IMP, imipenem; CFT, cefoxitin; AZT, aztreonam; CIP, ciprofloxacin; CTX, cefotaxime; TGC, tigecycline.

dra, sfa/foc, iutA, and kpsMT II $)^{31}$. These virulence genes are generally distributed on the chromosome. While we found that four virulence genes $(h l y F, o m p T$, sitA, and traT) are located on a plasmid containing an IncFII and an IncFIB replicon (designated p4_SCLZR49). These virulence genes were previously reported to be associated with virulence in avian-pathogenic E. coli strains. ${ }^{32,33}$ p4_SCLZR49 is 99,033 bp in length with an average GC content of $50.46 \%$ and encodes 115 predicted ORFs, including a tetracycline-resistant gene $\operatorname{tet}(A)$. A fullplasmid BLAST comparative analysis showed that p4_SCLZR49 exhibited high similarity $(>99.9 \%$ identity, $99 \%$ coverage) to $151-\mathrm{kb}$ pCombat13F7-2 (GenBank accession no. CP019247) and 392-kb pP2-3T (GenBank accession no. MG014722) from $E$. coli, which were recovered from a clinical patient in Hong Kong and a diseased pig in Guangzhou, in China, respectively. This result showed that almost the whole plasmid of p4_SCLZR49 is contained in pCombat13F7-2 and pP2-3T. Therefore, p4_SCLZR49 might be a fundamental virulence plasmid and potentially serves as an environmental reservoir for virulence genes $h l y F$, ompT, sitA, and traT, based on which hybrid plasmids harboring both virulence and multidrugresistant genes could be formed, like the cases of pCombat13F7-2 and pP2-3T (Figure 1).

K. pneumoniae SCLZR50 carried 50 different virulence genes (Table S1), including gene clusters contributing to formation of type 3 fimbriae for biofilm formation ( $m r k A B C D F I J H)$ and type 1 fimbriae for adherence (fimADEFGHI), mediating synthesis of enterobactin siderophore for iron acquisition (entABCDEF and fep $A B C D G$ ), as well as genes encoding T6SS (tssFGJ) and the ferric aerobactin receptor (iutA). Nineteen different virulence genes were identified in C. braakii SCLZR53 (Table S1). Among them, genes encoding biosynthesis and export of Vi polysaccharide (tviCDE and vexABCD), and type VI secretion system (tssCFM) made up the bulk of the virulence gene profiles. Additionally, E. coli group 3 capsule synthesis genes $k p s E$ and $k p s M_{-} K 11,{ }^{34}$ were detected on an 87-kb hybrid plasmid containing IncFIA, IncFIB, and IncY replicons (designated p6_SCLZR53) in SCLZR53.

\section{Phylogenetic Analysis of NDM5-Producing Isolates}

To determine a possible clinical relevance of CPE cultured from the aquatic environment, these three NDM5-producing strains were subjected to phylogenetic analysis. In silico analysis assigned SCLZR49 to ST1771, with an allelic profile 224-4-54-247-11-1-7 corresponding to the 7 housekeeping genes $a d k$, fum $C, \operatorname{gyr} B, i c d, m d h$, purA, and recA. The ST was primarily isolated from livestock and retail meats, ${ }^{35,36}$ and also detected in human and environmental samples. A core genome-based phylogeny for SCLZR49 and eighteen additional ST1771 isolates retrieved from EnteroBase identified a total of 3780 core genes with 9730 SNPs. Phylogenetic analysis revealed that SCLZR49 was present in a cluster with isolates of different origin (wild animals, livestock and poultry, and environment) from foreign countries (United States, Denmark, Mongolia, and Kenya) (Figure 2). Whereas SCLZR49 was a little less related to others within the clade and was closest to strain 19OR04PC05-EC (ESC_NA2556AA, carrying no carbapenemase gene, recovered from swine in 2019, United States), with 803 SNPs difference (Figure 2). Annotation of the phylogeny with $\beta$-lactamase gene carriage showed a diverse pattern of resistance genes and a low prevalence 
Table 2 Summary of the Features Associated with the Three NDM-5-Producing Enterobacteriaceae Strains

\begin{tabular}{|c|c|c|c|c|c|c|}
\hline Isolate & $\begin{array}{l}\text { Chromosome/ } \\
\text { Plasmid/Contig }\end{array}$ & $\begin{array}{l}\text { Length } \\
\text { (bp) }{ }^{a}\end{array}$ & $\begin{array}{l}\text { GC } \\
\%\end{array}$ & MLST & $\begin{array}{l}\text { Inc Type } \\
\left(p M L S T^{b}\right)\end{array}$ & Drug Resistance Gene \\
\hline \multirow{5}{*}{$\begin{array}{l}\text { SCLZR49 } \\
\text { (E. coli) }\end{array}$} & Chromosome & $4,883,356$ & 50.78 & STI77I & & $m d f(A)$ \\
\hline & pNDM5_SCLZR49 & 92,443 & 53.03 & - & IncR; IncXI & $\begin{array}{l}\operatorname{aadA2} \text { aph(3")-Ib; aph(3')-Ila; aph(6)-Id; rmtB; bla }{ }_{\mathrm{NDM}-5} ; \\
\text { bla }_{\mathrm{TEM}-\mathrm{IB}} ; m p h(A) ; \text { floR; oqxA; oqxB; sull; sul2; tet(A); dfrAI2 }\end{array}$ \\
\hline & PI_SCLZR49 & 210,840 & 46.97 & - & $\begin{array}{l}\text { IncHI2; } \\
\text { IncHI2A } \\
\left(\mathrm{ST}^{\mathrm{b}}\right)\end{array}$ & 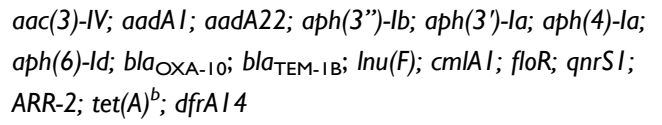 \\
\hline & P2_SCLZR49 & 3885 & 51.66 & - & Coll56 & \\
\hline & P4_SCLZR49 & 99,033 & 50.46 & - & $\begin{array}{l}\text { IncFIB; } \\
\text { IncFIl (F24: } \\
\text { A-:BI) }\end{array}$ & $\operatorname{tet}(A)$ \\
\hline \multirow{5}{*}{$\begin{array}{l}\text { SCLZR50 } \\
\text { (K. pneumoniae) }\end{array}$} & Chromosome & $5,089,532$ & 57.65 & ST5828 & - & $b^{\prime} a_{\mathrm{SHV}-26} ;$ fos $A 5 ;$ oqxA; oqx $B$ \\
\hline & pNDM5_SCLZR50 & 53,134 & 47.35 & - & $\operatorname{lnc} \times 3$ & $r m t B ; b l a_{\mathrm{NDM}-5} ; b l a_{\mathrm{TEM}-\mathrm{IB}} ;$ fosA3 \\
\hline & PI_SCLZR50 & 102,920 & 49.76 & - & $\begin{array}{l}\operatorname{lncFIB}(\mathrm{K}) \\
\text { (F-:A-:B-); } \\
\text { IncR }\end{array}$ & \\
\hline & P2_SCLZR50 & 132,778 & 52.32 & - & $\begin{array}{l}\text { IncFII(K) } \\
(\mathrm{K} 2: \mathrm{A}-\mathrm{B}-)\end{array}$ & 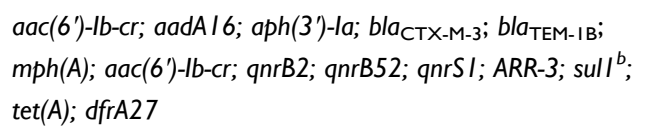 \\
\hline & P3_SCLZR50 & 248,272 & 47.22 & - & $\begin{array}{l}\text { IncHIIB } \\
\text { (UT) }\end{array}$ & 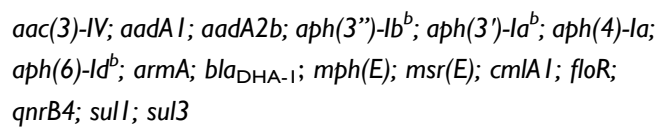 \\
\hline \multirow{7}{*}{$\begin{array}{l}\text { SCLZR53 } \\
\text { (C. braakii) }\end{array}$} & Chromosome & $5,263,019$ & 51.89 & - & - & $b^{\prime} a_{C M Y-101}$ \\
\hline & pNDM5_SCLZR53 & 52,041 & 47.58 & - & $\operatorname{lnc} \times 3$ & $r m t B ; b l a_{\mathrm{NDM}-5} ; b l a_{\mathrm{TEM}-\mathrm{IB}} ;$ fos $A 3$ \\
\hline & PI_SCLZR53 & $\sim 22,835^{a}$ & 50.37 & - & UT & \\
\hline & P2_SCLZR53 & $\sim 286,080$ & 48.91 & - & $\begin{array}{l}\text { IncHI2; } \\
\text { IncHI2A } \\
\left(\mathrm{ST}^{\mathrm{b}}\right) \text {; } \\
\text { IncN; IncR }\end{array}$ & 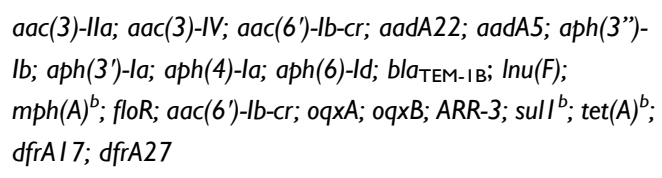 \\
\hline & P4_SCLZR53 & 56,447 & 52.68 & - & UT & \\
\hline & p5_SCLZR53 & $\sim 62,677^{a}$ & 34.08 & - & UT & \\
\hline & p6_SCLZR53 & 87,197 & 50.53 & - & $\begin{array}{l}\operatorname{lncFIA}(H I I) \\
\text { IncFIB(K) } \\
\text { (F-:AI4:B-); } \\
\text { IncY }\end{array}$ & \\
\hline
\end{tabular}

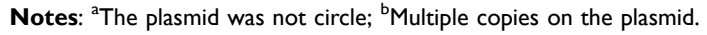

Abbreviations: -, not available; UT, unknown type.

of carbapenemase genes in the characterized isolates, with SCLZR49 being the only strain carrying the carbapenemase gene $b l a_{\mathrm{NDM}-5}$ (Figure 2).
MLST analysis revealed that $K$. pneumoniae SCLZR50 did not belong to any known ST. It belongs to the novel ST5828 (gapA-infB-mdh-pgi-phoE-rpoB-tonB allele number 
2-3-6-1-16-7-4) and its nearest sequence types are ST34 (gapA-infB-mdh-pgi-phoE-rpoB-tonB, 2-3-6-1-9-7-4) and ST1444 (gapA-infB-mdh-pgi-phoE-rpoB-tonB, 2-3-6-1-17$7-4)$, which were mainly isolated clinically. Twenty-six whole-genome sequences of ST34 strains and one from an ST1444 strain retrieved from GenBank were aligned with that of strain SCLZR50. Three thousand seventy-nine core genes were shared by these genome sequences with 43,782 SNP. These $K$. pneumoniae strains were mainly clinical isolates from around the world. SCLZR50 was closely genetically related, with 3032 SNPs, to an IMP-4 producing clinical isolate XPY193 (GCF_ 002740705.1) from a patient with bloodstream infection in China in 2016 (Figure 3). Resistance gene profiles showed a native $\beta$ lactamase gene $b l a_{\mathrm{SHV}-26}$ and a sporadical acquisition of different kinds of carbapenemase genes, including $b l a_{\mathrm{NDM}-5}, b l a_{\mathrm{NDM}-7}, b_{\mathrm{KPC}-2}, b_{a_{\mathrm{KPC}-3}}$, bla $_{\mathrm{IMP}-1}, b a_{\mathrm{IMP}-4}$, and $b l a_{\mathrm{OXA}-181}$ in these characterized isolates (Figure 3).

Phylogenetic analysis based on core genome alignment of SCLZR53 and 50 publicly $C$. braakii strains retrieved from GenBank (on 2020/10/13) showed a diverse set of genomes of 2598 core genes with around 300, 000 SNPs. These $C$. braakii isolates were widely found in the environment and in clinical specimens across various countries (Figure 4). SCLZR53 had the most closely genetic relationship with environmental isolates $\mathrm{C} 8$ (GCF 008364635.1, recovered in 2019 in Germany) and HH9 (GCF_ 004331635.1, recovered in 2019 in Canada), with 17,073 and 17,005 SNPs differences, respectively (Figure 4). Resistance genes presented on all C. braakii isolates showed a common $\beta$-lactamase gene $b l a_{\mathrm{CMY}}$ and independent acquisitions of carbapenemase genes including $b l a_{\mathrm{NDM}-5}$, bla $a_{\mathrm{KPC}-2}$, and $b l a_{\mathrm{OXA}-48}$ (Figure 4).

\section{NDM5-Encoding Plasmids}

bla $a_{\mathrm{NDM}-5}$ gene identified in this study was located on a 92,443-bp IncR/IncX1 plasmid (pNDM5_SCLZR49) in E. coli, a 53,134-bp IncX3 plasmid (pNDM5_SCLZR50) in $K$. pneumoniae and a 52,041-bp IncX3 plasmid (pNDM5_SCLZR53) in C. braakii, respectively (Table 2).

pNDM5_SCLZR49 had an average GC content of $53.03 \%$ and contained 102 ORFs, including 15 resistance genes. Sequence analysis of pNDM5_SCLZR49 presented a hybrid structure consisting of a 9.4-kb IncR backbone (nt 1 to 7584 bp, nt 90,590 to 92,443 bp) which was almost identical (99\% coverage, $99.2 \%$ identity) to the plasmid pKPS77 (GenBank accession no. KF954150) from a clinical K. pneumoniae strain in France, ${ }^{37}$ and a $8.9-\mathrm{kb}$
IncX1 backbone (nt 46,263 to 56,289 bp) which was similar to (91\% coverage, $99.69 \%$ identity) the plasmid pOLA52 (GenBank accession no. EU370913) from E. coli recovered from swine manure in Denmark ${ }^{38}$ (Figure 5). $b l a_{\mathrm{NDM}-5}$ was located within a $11.0-\mathrm{kb}$ IS26-bounded region consisting of a 286-bp remnant of ISAba125, bla $a_{\mathrm{NDM}-5}$, ble (mediating bleomycin resistance), $\operatorname{trpF}$ (encoding a phosphoribosyl anthranilate isomerase), $d s b C$ (encoding an oxidoreductase), tnp $A$ IS91 and a class 1

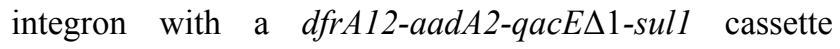
array, which was truncated by IS 26 at its $5^{\prime}$ conserved segment (Figure 5). The same $b l a_{\mathrm{NDM}-5}$ carrying structure was also identified in an IncFII plasmid pNDM-SDCRK18 -7 (Accession no. MN641485) from E. coli in China in 2019 (Figure 5). It is likely that pNDM5_SCLZR49 acquires the $b l a_{\mathrm{NDM}-5}$ region through IS26-mediated homologous recombination, as no characteristic 8-bp direct target repeats were observed flanking the IS26bracketed region. Sequence alignment showed that pNDM5_SCLZR49 shared 99.76\% nucleotide identity (95\% coverage) with a $116.8-\mathrm{kb}$ plasmid p8C57-NDM (Accession no. MT407546) identified in an E. coli isolate from chicken in Guangdong, China in 2020.

Analysis of the gene organization revealed that pNDM5_SCLZR50 and pNDM5_SCLZR53 were almost identical, with a deletion of a truncated ISAba125 fragment between IS5 and IS3000 in pNDM5_SCLZR53 representing the only modular difference (Figure 6). The two identified plasmids exhibited high similarity with $>90 \%$ coverage and $>99.9 \%$ identity to several previously reported $b l a_{\mathrm{NDM}}$-carrying IncX3 plasmids in humans, ${ }^{39,40}$ including the epidemic $b l a_{\mathrm{NDM}-5}$-carrying plasmid pNDMMGR194 (Accession no. KF220657) identified in $K$. pneumoniae in India (Figure 6). The $b l a_{\mathrm{NDM}-5}$ regions

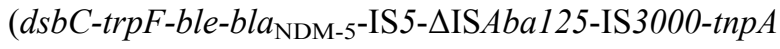

Tn2) of pNDM5_SCLZR50 and pNDM-MGR194 were identical. While, a 7.7-kb IS26-bounded multi-drug resistant (MDR) region, containing ARGs fos $A 3, r m t B$, and $b l a_{\mathrm{TEM}-1 \mathrm{~B}}$, was present upstream of the $b l a_{\mathrm{NDM}-5}$ region in pNDM5_SCLZR50. It was likely that homologous recombination between these two copies of IS26 resulted in the deletion of MDR region in pNDM-MGR194 (Figure 6). Alignment analysis of pNDM5_SCLZR50 and pNDM-HN380 showed that the genetic context of $b l a_{\mathrm{NDM}-5}$ is highly similar to that of $b l a_{\mathrm{NDM}-1}$, with the exception of an inversion of IS5 downstream of bla $a_{\mathrm{NDM}}$ (Figure 6). Whereas, there was a completely different MDR region in these two plasmids, which most likely 


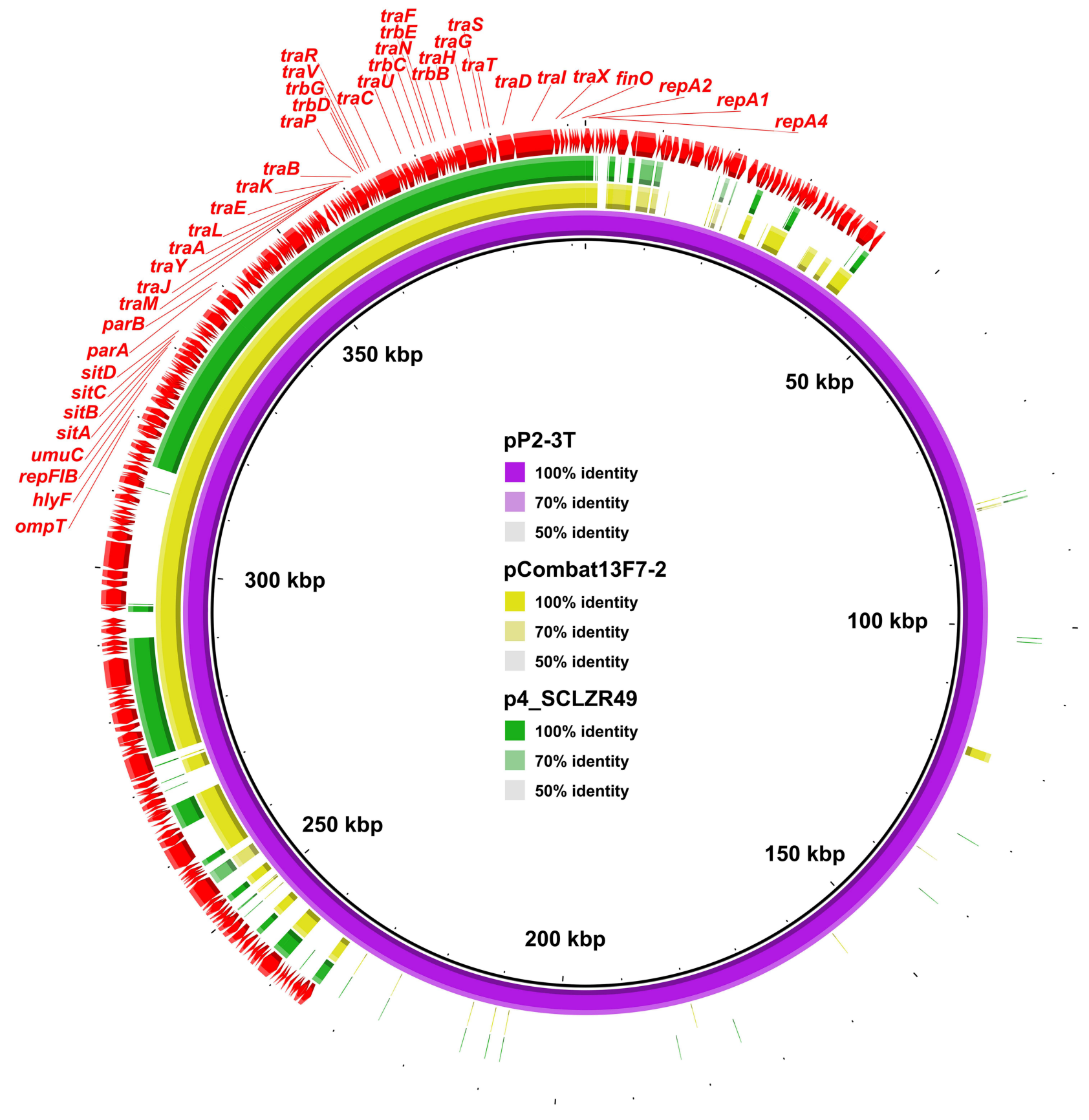

Figure I Comparison of the plasmid p4_SCLZR49 with pCombat I3F7-2 and pP2-3T. pP2-3T was used as a reference to compare with other plasmids. Gaps in the circular maps refer to plasmid regions that were missing in the respective plasmid compared to the reference plasmid.

arose from multiple insertion and homologous recombination events of IS26. This result suggested that pNDM5_SCLZR50 did not emerge from sequential mutations of a pNDM-HN380-like plasmid carrying bla $a_{\mathrm{NDM}-1}$,

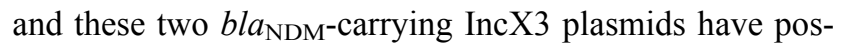
sibly evolved with different genetic patterns.

Conjugation experiments showed that $b l a_{\mathrm{NDM}-5}$ genes in the three isolates could be successfully transferred to E. coli
J53, respectively. Compared with the recipient strain J53, transformants carrying $b l a_{\mathrm{NDM}-5}$ exhibited significantly increased resistance to carbapenems and cephalosporins, suggesting that $b l a_{\mathrm{NDM}-5}$ genes in donor strains were functional. The results also suggested that pNDM5_SCLZR50 and pNDM5_SCLZR53 were self-transmissible. For the lack of transfer regions for conjugation, pNDM5_SCLZR49 might be mobilizable in the presence of a helper plasmid. 


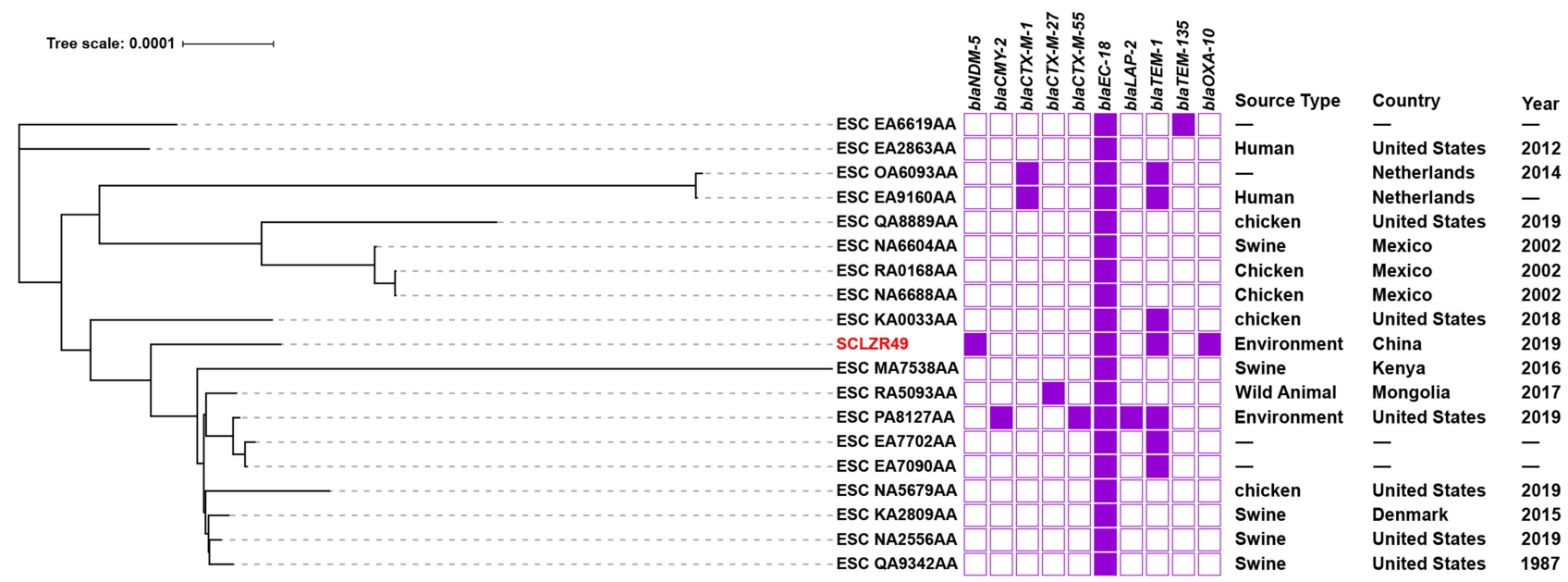

Figure 2 A phylogenetic analysis of the core genomes of E. coli SCLZR49 identified in this study and I8 additional STI77I isolates retrieved from EnteroBase. SCLZR49 is indicated in red. The presence or absence of antibiotic resistance genes is indicated by filled or empty squares, respectively. The tree scale indicates substitutions per site. Abbreviation: -, not available.

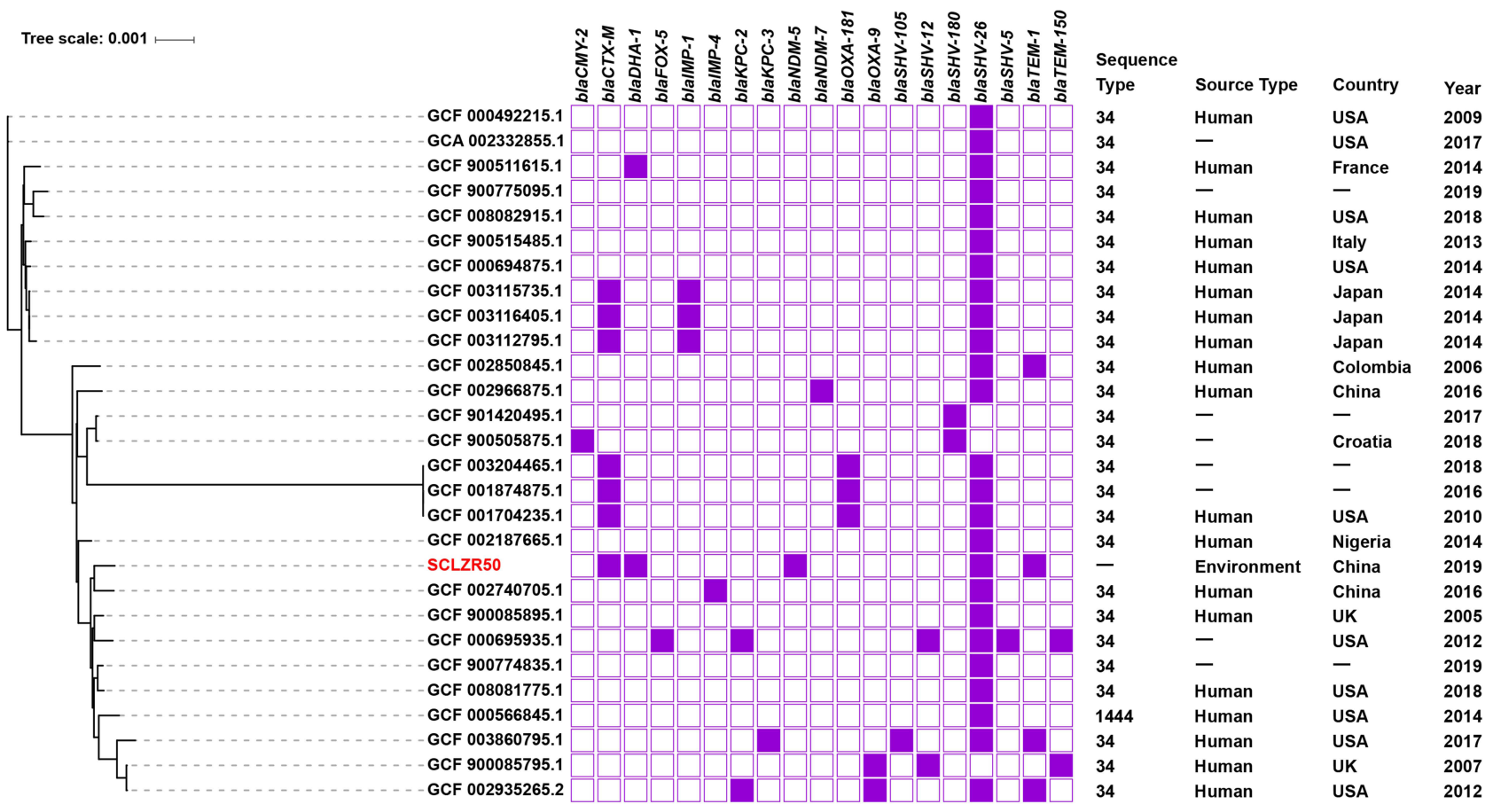

Figure 3 A phylogenetic analysis of the core genomes of K. pneumoniae SCLZR50 identified in this study and 27 additional K. pneumoniae strains with adjacent sequence type available from GenBank. SCLZR50 is indicated in red. The presence or absence of antibiotic resistance genes is indicated by filled or empty squares, respectively. The tree scale indicates substitutions per site.

Abbreviations: -, not available.

\section{Discussion}

$\mathrm{CPE}$ are listed by the $\mathrm{WHO}$ as critically important priority list of pathogens, as infections caused by CPE are extremely difficult to manage due to the limited treatment options available. ${ }^{41}$ In recent years, clinically relevant CPE are widely documented in the natural environment. ${ }^{16,42,43}$ Among them, the urban river is of particular importance because it represents a most basic resource for urban residents and it is a realistic possibility for transmission of CPE from contaminated water bodies to humans through water contact.

Previous studies showed that $b l a_{\mathrm{NDM}}$ genes were prevalent in river systems, especially the gene subtype $b l a_{\mathrm{NDM}-1},{ }^{10,15}$ and detection of $b l a_{\mathrm{NDM}-5}$ in river waters 

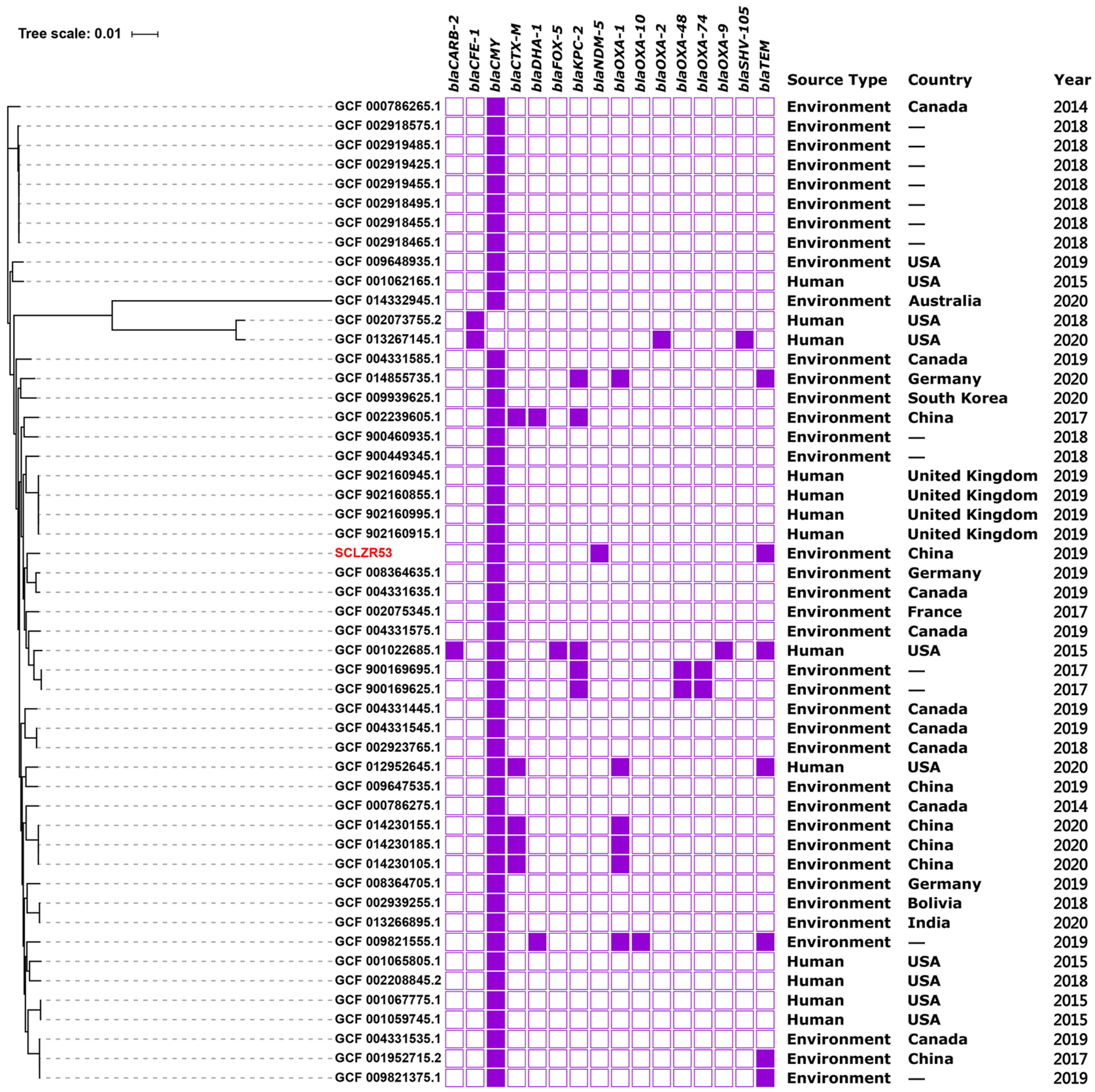

Figure 4 A phylogenetic analysis of the core genomes of C. braakii SCLZR53 identified in this study and 50 additional C. braakii strains available from GenBank. SCLZR53 is indicated in red. The presence or absence of antibiotic resistance genes is indicated by filled or empty squares, respectively. The tree scale indicates substitutions per site. Abbreviation: -, not available.

was primarily associated with E. coli. ${ }^{12,18}$ In this study, $b l a_{\mathrm{NDM}-5}$ was detected in three different bacterial species (E. coli, K. pneumoniae, and C. braakii) of Enterobacteriaceae in an urban river in China. These three $b l a_{\mathrm{NDM}-5}$-harboring isolates were multi-drug resistant (acquired resistance to three or more classes of antibiotics tested) ${ }^{44}$ and contained a large number of ARGs that are carried primarily by plasmids. The presence and dissemination of clinically significant ARGs in the environmental water represent a cause of concern for public health. Virulence gene profiling revealed that they harbored many genes associated with pathogenicity, colonization and survival in the host, implying the virulence potential of $b l a_{\mathrm{NDM}-5}$-harboring isolates in this study. The detection of a plasmid carrying E. coli group 3 capsule synthesis genes $k p s E$ and $k p s M_{-} K 11$ in C. braakii SCLZR53 suggested a possible horizontal transfer of virulence genes between bacteria of different 


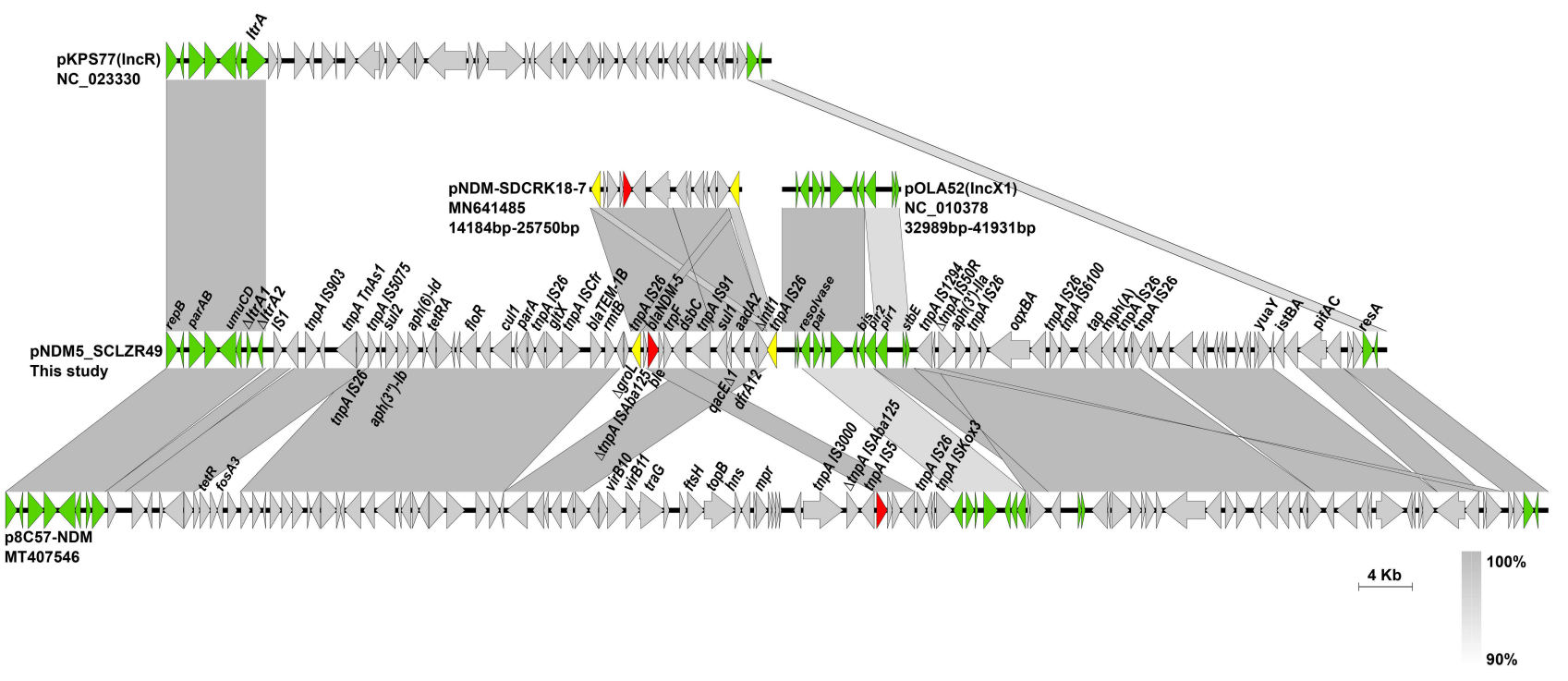

Figure 5 Comparison of linear maps of PNDM5_SCLZR49 and other closely related plasmids. Genes and insertion sequences are indicated by arrows and $\triangle$ indicates the truncated gene. bla $a_{\mathrm{NDM}-5}$ is indicated in red and backbone regions are in green. Gray shades denote shared regions with a high degree of homology.

species in the environment. The identification of virulence genes in plasmids in this study highlights the potential of virulence determinants to be further disseminated into human, which poses a big challenge to public health.
Bacterial antimicrobial resistance is constantly evolving and plasmids play a major role in the horizontal spread of ARGs between multiple species and strains. ${ }^{45}$ The identification of plasmid characteristics is essential to gain a better understanding of the transmission and mole-

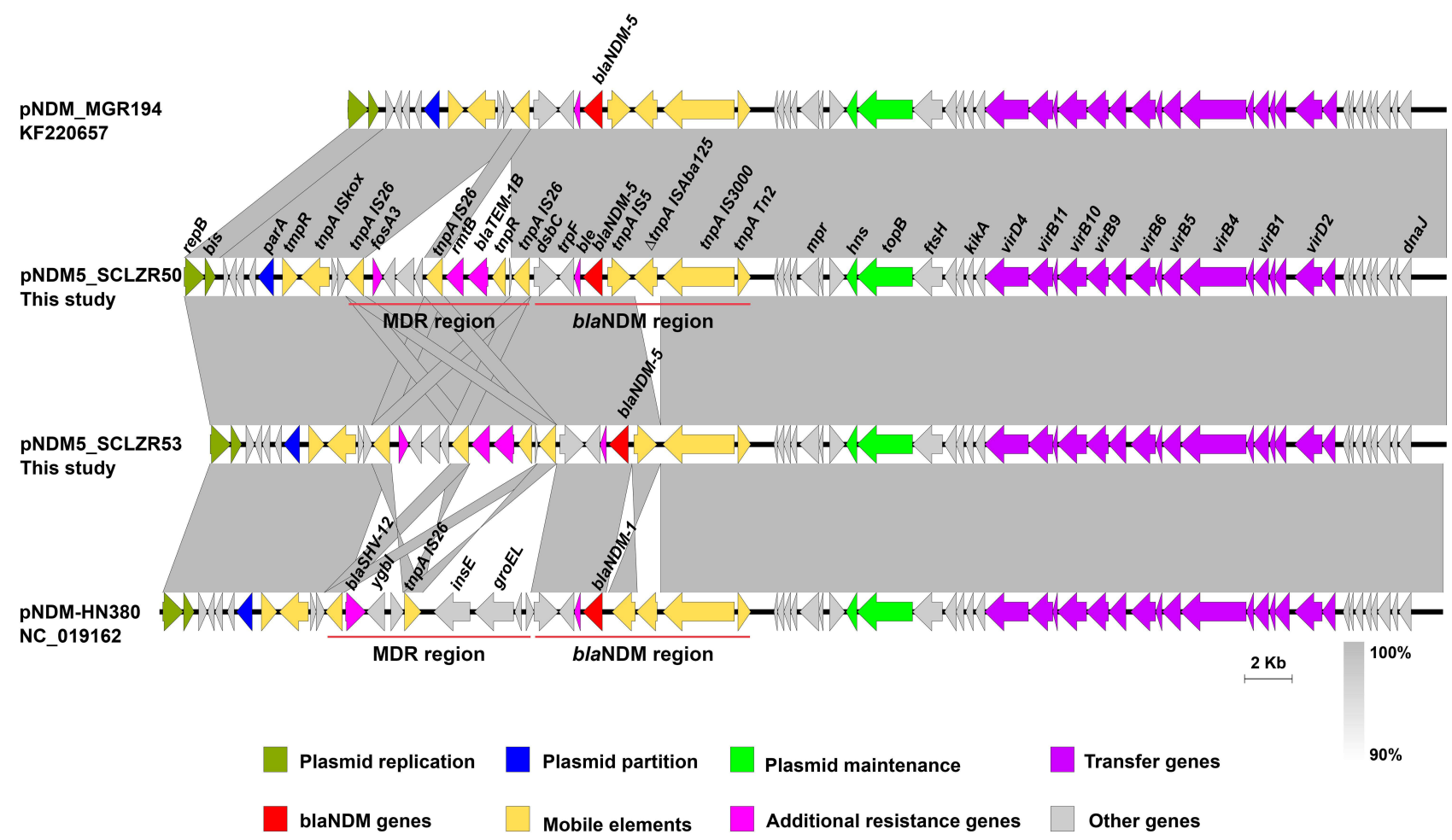

Figure 6 Comparison of linear maps of pNDM5_SCLZR50, pNDM5_SCLZR53, pNDM-HN380, and pNDM-MGRI94. Genes and insertion sequences are indicated by arrows and $\Delta$ indicates the truncated gene. Gray shades denote shared regions with a high degree of homology. 
cular epidemiology of ARGs. In this study, three NDM5-encoding plasmids revealed two different patterns of dissemination of $b l a_{\mathrm{NDM}-5}$. pNDM5_SCLZR49 in E. coli represented an example of the emergence of a novel plasmid structure harboring $b l a_{\mathrm{NDM}-5}$. Molecular identification of pNDM5_SCLZR49 revealed recombination of genetic contents from different plasmids, highlighting a high genetic plasticity of plasmid vectors. By integration of multiple replicons, such a novel hybrid plasmid, like pNDM5_SCLZR49, may be adopted by more bacterial hosts from different ecological niches, thereby conferring a rapid accumulation and dissemination of clinically relevant ARGs. Several studies supported the key role of IncX3-type pNDM-MGR194-like plasmid as an efficient vector in the dissemination of $b l a_{\mathrm{NDM}-5}$ among Enterobacteriaceae isolates from both inside and outside of clinical settings. ${ }^{4,39,46}$ A recent study by Zhao et al demonstrated that isolates harboring closely related or identical bla NDM-5- carrying IncX3 plasmids transmit across different environmental matrices and humans. ${ }^{14}$ The identification of IncX3 plasmids pNDM5_SCLZR50 and pNDM5_SCLZR53 in this study provided an additional evidence of this. Besides, high similarity between the two transferable bla $a_{\mathrm{NDM}-5}$-carrying plasmids, pNDM5_SCLZR50 in $K$. pneumoniae and pNDM5_SCLZR53 in C. braakii, pointed to a common source of these two plasmids, highlighting that the dissemination of $b l a_{\mathrm{NDM}-5}$ in aquatic environment is driven by epidemic plasmids to different hosts. In addition, genetic analysis of bla $a_{\mathrm{NDM}-5}$-haboring plasmids in this study showed clinically relevant, which poses a public health concern.

NDM-5 was first identified in an E. coli clinical isolate belonging to ST648 in the United Kingdom in 2011. ${ }^{47}$ In recent years, there has been widespread of NDM-5 in $K$. pneumoniae and $E$. coli with certain STs (for K. pneumoniae, ST14, ST16, ST23 or ST147; for E. coli, ST167 or ST410), ${ }^{48-54}$ with ST23 and ST167 being the most common types of NDM-positive $K$. pneumoniae and E. coli strains, respectively, in China. ${ }^{4}$ While NDM-5 is most common in E. coli, ${ }^{4}$ E. coli ST1771 has not been reported in China before. The bla $a_{\mathrm{NDM}-5}$-harboring SCLZR49 in this study represents the first identification of $E$. coli ST1771 in this region, which further extends its role in enhancing the spread of $b l a_{\mathrm{NDM}-5}$. K. pneumoniae is the most common host of bla $_{\mathrm{NDM}}{ }^{4}{ }^{4} l a_{\mathrm{NDM}-5}$-positive $K$. pneumoniae is frequently reported in humans ${ }^{55,56}$ and animals, ${ }^{57}$ while rarely in aquatic environment in China. Recently, Zhao et al reported a K. pneumoniae ST229 harboring $b l a_{\mathrm{NDM}-5}$ recovered from river water in eastern China. $^{14}$ This study identified a bla $a_{\mathrm{NDM}-5}$-carrying $K$. pneumoniae isolate belonging to a new ST in Southwest China, reinforcing the important host role of $K$. pneumoniae in the spread of $b l a_{\mathrm{NDM}-5}$ in diverse ecological niches. bla $a_{\mathrm{NDM}}$-harboring Citrobacter spp. were sporadically reported in China, including an NDM-5-producing $C$. freundii from hospitalized patient $^{39}$ and

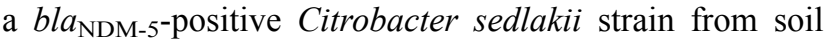
in an intensive vegetable cultivation area. ${ }^{14}$ To our knowledge, this is the first time to identify a $b l a_{\mathrm{NDM}-5}$-harboring C. braakii, which further increases the host spectrum of this important resistance gene.

This study has several limitations. The number of isolates characterized is limited and the isolates were only obtained from two sampling sites. The study does not represent the prevalence of CPE in Tuojiang River for the limited number of samples collected in a short period. Periodic sampling at the same and additional sites is needed to uncover the dynamics of dissemination of CPE in Tuojiang River.

\section{Conclusion}

In this study, we characterized three $b l a_{\mathrm{NDM}-5}$-harboring Enterobacteriaceae isolates from an urban river by whole-genome analysis. These NDM-5- producing isolates carry numerous antibiotic resistance genes and virulence genes, hinting at a possible contribution of these strains to spread carbapenem resistance as well as to cause antibiotic-resistant infectious diseases. Although identification of these bla $a_{\mathrm{NDM}-5}$-harboring isolates as clinically relevant strains could not be demonstrated in the current study, the close genetic relationship between their $b l a_{\mathrm{NDM}-5}$ plasmids with those from clinical strains suggested the possibility of plasmid transmission from clinical settings to aquatic environment through horizontal gene transfer events. Taken together, this study points to the fact that carbapenemase gene $b l a_{\mathrm{NDM}-5}$ is an important pollutant of river ecosystems, highlighting the great need to monitor for CPE in the environment and their epidemiological links with humans.

\section{Acknowledgments}

This work was supported by National Natural Science Foundation of China (31900125), the Project supported by the Joint Funds of the Luzhou and Southwest Medical 
University Natural Science Foundation (2019LZXNYDJ47), and Natural Science Foundation of Southwest Medical University (2020ZRQNA022). The funders had no role in study design, data collection and interpretation, or the decision to submit the work for publication.

\section{Disclosure}

The authors report no conflicts of interest in this work.

\section{References}

1. Nordmann P, Dortet L, Poirel L. Carbapenem resistance in Enterobacteriaceae: here is the storm! Trends Mol Med. 2012;18 (5):263-272. doi:10.1016/j.molmed.2012.03.003

2. van Duin D, Doi Y. The global epidemiology of carbapenemase-producing Enterobacteriaceae. Virulence. 2017;8 (4):460-469. doi:10.1080/21505594.2016.1222343

3. Partridge SR. Resistance mechanisms in Enterobacteriaceae. Pathology. 2015;47(3):276-284. doi:10.1097/pat.0000000000000237

4. Wu W, Feng Y, Tang G, Qiao F, McNally A, Zong Z. NDM metallo$\beta$-lactamases and their bacterial producers in health care settings. Clin Microbiol Rev. 2019;32(2):e00115-18. doi:10.1128/CMR

5. Karkman A, Do TT, Walsh F, Virta MPJ. Antibiotic-resistance genes in waste water. Trends Microbiol. 2018;26(3):220-228. doi:10.1016/j. tim.2017.09.005

6. Sun P, Bi Z, Nilsson M, et al. Occurrence of $b l a_{\mathrm{KPC}-2}, b l a_{\mathrm{CTX}-\mathrm{M}}$, and $m c r-1$ in Enterobacteriaceae from well water in rural China. Antimicrob Agents Chemother. 2017;61(4). doi:10.1128/AAC.02569-16

7. Xu G, Jiang Y, An W, Wang H, Zhang X. Emergence of KPC-2-producing Escherichia coli isolates in an urban river in Harbin, China. World J Microbiol Biotechnol. 2015;31 (9):1443-1450. doi:10.1007/s11274-015-1897-Z

8. Yang F, Huang L, Li L, Yang Y, Mao D, Luo Y. Discharge of KPC-2 genes from the WWTPs contributed to their enriched abundance in the receiving river. Sci Total Environ. 2017;581-582:136-143. doi:10.1016/j.scitotenv.2016.12.063

9. Yang F, Gu Y, Zhou J, Zhang K. Swine waste: a reservoir of high-risk bla $_{\mathrm{NDM}}$ and mcr-1. Sci Total Environ. 2019;683:308-316. doi:10.1016/j.scitotenv.2019.05.251

10. Yang F, Mao D, Zhou H, Wang X, Luo Y. Propagation of New Delhi Metallo- $\beta$-lactamase genes $\left(b l a_{\mathrm{NDM}-1}\right)$ from a wastewater treatment plant to its receiving river. Environ Sci Tech Let. 2016;3(4):138-143. doi:10.1021/acs.estlett.6b00036

11. Zhang R, Li J, Wang Y, Shen J, Shen Z, Wang S. Presence of NDM in non-E. coli Enterobacteriaceae in the poultry production environment J Antimicrob Chemother. 2019;74(8):2209-2213. doi:10.1093/jac/dkz193

12. Bleichenbacher S, Stevens MJA, Zurfluh K, et al. Environmental dissemination of carbapenemase-producing Enterobacteriaceae in rivers in Switzerland. Environ Pollut. 2020;265:115081. doi:10.1016/j. envpol.2020.115081

13. Yang J, Wang H, Roberts DJ, et al. Persistence of antibiotic resistance genes from river water to tap water in the Yangtze River Delta. Sci Total Environ. 2020;742:140592. doi:10.1016/j. scitotenv.2020.140592

14. Zhao Q, Berglund B, Zou H, et al. Dissemination of bla $a_{\mathrm{NDM}-5}$ via IncX3 plasmids in carbapenem-resistant Enterobacteriaceae among humans and in the environment in an intensive vegetable cultivation area in eastern China. Environ Pollut. 2021;273:116370. doi:10.1016/ j.envpol.2020.116370

15. Suzuki Y, Nazareno PJ, Nakano R, et al. Environmental presence and genetic characteristics of carbapenemase-producing Enterobacteriaceae from hospital sewage and river water in the Philippines. Appl Environ Microbiol. 2020;86(2). doi:10.1128/AEM.01906-19
16. Teixeira $\mathrm{P}$, Tacao $\mathrm{M}$, Pureza $\mathrm{L}$, et al. Occurrence of carbapenemase-producing Enterobacteriaceae in a Portuguese river: bla $a_{\mathrm{NDM}}, b l a_{\mathrm{KPC}}$ and $b l a_{\mathrm{GES}}$ among the detected genes. Environ Pollut. 2020;260:113913. doi:10.1016/j.envpol.2020.113913

17. Piedra-Carrasco N, Fabrega A, Calero-Caceres W, et al. Carbapenemase-producing Enterobacteriaceae recovered from a Spanish river ecosystem. PLoS One. 2017;12(4):e0175246. doi:10.1371/journal.pone. 0175246

18. Almakki A, Maure A, Pantel A, et al. NDM-5-producing Escherichia coli in an urban river in Montpellier, France. Int J Antimicrob Agents. 2017;50(1):123-124. doi:10.1016/j.ijantimicag.2017.04.003

19. Di DYW, Jang J, Unno T, Hur H-G. Emergence of Klebsiella variicola positive for NDM-9, a variant of New Delhi metallo- $\beta$ lactamase, in an urban river in South Korea. $J$ Antimicrob Chemother. 2017;dkw547. doi:10.1093/jac/dkw547

20. Tafoukt R, Touati A, Leangapichart T, Bakour S, Rolain JM. Characterization of OXA-48-like-producing Enterobacteriaceae isolated from river water in Algeria. Water Res. 2017;120:185-189. doi:10.1016/j.watres.2017.04.073

21. Zhang L, Ma X, Luo L, et al. The prevalence and characterization of Extended-Spectrum beta-Lactamase- and carbapenemase-producing bacteria from hospital sewage, treated effluents and receiving rivers. Int J Environ Res Public Health. 2020;17(4). doi:10.3390/ijerph17041183

22. Lane DJ. 16S/23S rRNA sequencing. Nucleic Acid Techniques Bacterial Syst. 1991;1:115-175.

23. Poirel L, Walsh TR, Cuvillier V, Nordmann P. Multiplex PCR for detection of acquired carbapenemase genes. Diagn Microbiol Infect Dis. 2011;70(1):119-123. doi:10.1016/j.diagmicrobio.2010.12.002

24. Institute CLS. Performance Standards for Antimicrobial Susceptibility Testing. Clinical and Laboratory Standards Institute Wayne, PA, USA; 2017.

25. Giske CG, Turnidge J, Canton R, Kahlmeter G. Update From The European Committee On Antimicrobial Susceptibility Testing (EUCAST). J Clin Microbiol. 2021;21:JCM0027621.

26. Seemann T. Prokka: rapid prokaryotic genome annotation. Bioinformatics. 2014;30(14):2068-2069. doi:10.1093/bioinformatics/btu153

27. Siguier P, Perochon J, Lestrade L, Mahillon J, Chandler M. ISfinder: the reference centre for bacterial insertion sequences. Nucleic Acids Res. 2006;34(Databaseissue):D32-D36. doi:10.1093/nar/gkj014

28. Alikhan NF, Petty NK, Ben Zakour NL, Beatson SA. BLAST Ring Image Generator (BRIG): simple prokaryote genome comparisons. BMC Genomics. 2011;12:402. doi:10.1186/14712164-12-402

29. Page AJ, Taylor B, Delaney AJ, et al. SNP-sites: rapid efficient extraction of SNPs from multi-FASTA alignments. Microb Genom. 2016;2(4):e000056. doi:10.1099/mgen.0.000056

30. Hu Y, Feng Y, Qin J, Zhang X, Zong Z. Acinetobacter chinensis, a novel Acinetobacter species, carrying $b l a_{\mathrm{NDM}-1}$, recovered from hospital sewage. J Microbiol. 2019;57(5):350-355. doi:10.1007/ s12275-019-8485-0

31. Johnson JR, Murray AC, Gajewski A, et al. Isolation and molecular characterization of nalidixic acid-resistant extraintestinal pathogenic Escherichia coli from retail chicken products. Antimicrob Agents Chemother. 2003;47(7):2161-2168. doi:10.1128/aac.47.7.21612168.2003

32. Rodriguez-Siek KE, Giddings CW, Doetkott C, Johnson TJ, Nolan LK. Characterizing the APEC pathotype. Vet Res. 2005;36 (2):241-256. doi:10.1051/vetres:2004057

33. Murase K, Martin P, Porcheron G, et al. HlyF produced by extraintestinal pathogenic Escherichia coli is a virulence factor that regulates outer membrane vesicle biogenesis. J Infect Dis. 2016;213 (5):856-865. doi:10.1093/infdis/jiv506

34. Steenbergen SM, Vimr ER. Biosynthesis of the Escherichia coli K1 group 2 polysialic acid capsule occurs within a protected cytoplasmic compartment. Mol Microbiol. 2008;68(5):1252-1267. doi:10.1111/ j.1365-2958.2008.06231.x 
35. Hao Y, Shao C, Bai Y, Jin Y. Genotypic and phenotypic characterization of IncX3 plasmid carrying bla NDM-7 in Escherichia coli sequence type 167 isolated from a patient with urinary tract infection. Front Microbiol. 2018;9:2468. doi:10.3389/fmicb.2018.02468

36. Adefioye OJ, Weinreich J, Rodiger S, Schierack P, Olowe OA. Phylogenetic characterization and multilocus sequence typing of extended-spectrum beta lactamase-producing Escherichia coli from food-producing animals, beef, and humans in southwest Nigeria. Microb Drug Resist. 2020. doi:10.1089/mdr.2019.0397

37. Compain F, Frangeul L, Drieux L, et al. Complete nucleotide sequence of two multidrug-resistant IncR plasmids from Klebsiella pneumoniae. Antimicrob Agents Chemother. 2014;58(7):4207-4210. doi:10.1128/aac.02773-13

38. Norman A, Hansen LH, She Q, Sorensen SJ. Nucleotide sequence of pOLA52: a conjugative IncX1 plasmid from Escherichia coli which enables biofilm formation and multidrug efflux. Plasmid. 2008;60 (1):59-74. doi:10.1016/j.plasmid.2008.03.003

39. Li X, Fu Y, Shen M, et al. Dissemination of bla $a_{\mathrm{NDM}-5}$ gene via an IncX3-type plasmid among non-clonal Escherichia coli in China. Antimicrob Resist Infect Control. 2018;7:59. doi:10.1186/s13756018-0349-6

40. Wang Y, Tong MK, Chow KH, et al. Occurrence of highly conjugative IncX3 epidemic plasmid carrying $b l a_{\mathrm{NDM}}$ in Enterobacteriaceae isolates in geographically widespread areas. Front Microbiol. 2018;9:2272. doi:10.3389/fmicb.2018.02272

41. Tacconelli E, Carrara E, Savoldi A, et al. Discovery, research, and development of new antibiotics: the WHO priority list of antibiotic-resistant bacteria and tuberculosis. Lancet Infect Dis. 2018;18(3):318-327. doi:10.1016/S1473-3099(17)30753-3

42. Gomi R, Matsuda T, Yamamoto M, et al. Characteristics of carbapenemase-producing Enterobacteriaceae in wastewater revealed by genomic analysis. Antimicrob Agents Chemother. 2018;62(5). doi:10.1128/AAC.02501-17

43. Mahon BM, Brehony C, Cahill N, et al. Detection of OXA-48-likeproducing Enterobacterales in Irish recreational water. Sci Total Environ. 2019;690:1-6. doi:10.1016/j.scitotenv.2019.06.480

44. Magiorakos AP, Srinivasan A, Carey RB, et al. Multidrug-resistant, extensively drug-resistant and pandrug-resistant bacteria: an international expert proposal for interim standard definitions for acquired resistance. Clin Microbiol Infect. 2012;18(3):268-281. doi:10.1111/ j.1469-0691.2011.03570.x

45. Rozwandowicz M, Brouwer MSM, Fischer J, et al. Plasmids carrying antimicrobial resistance genes in Enterobacteriaceae. J Antimicrob Chemother. 2018;73(5):1121-1137. doi:10.1093/jac/dkx488

46. Pak-Leung Ho YW. IncX3 epidemic plasmid carrying $b l a_{\mathrm{NDM}-5}$ in Escherichia coli from swine in multiple geographic areas in China. Antimicrob Agents Chemother. 2017;62(3):e02295-02217.
47. Hornsey M, Phee L, Wareham DW. A novel variant, NDM-5, of the New Delhi metallo-beta-lactamase in a multidrug-resistant Escherichia coli ST648 isolate recovered from a patient in the United Kingdom. Antimicrob Agents Chemother. 2011;55 (12):5952-5954. doi:10.1128/AAC.05108-11

48. Zhu YQ, Zhao JY, Xu C, Zhao H, Jia N, Li YN. Identification of an NDM-5-producing Escherichia coli sequence type 167 in a neonatal patient in China. Sci Rep. 2016;6:29934. doi:10.1038/srep29934

49. Xu L, Wang P, Cheng J, Qin S, Xie W. Characterization of a novel bla $_{\mathrm{NDM}-5}$-harboring IncFII plasmid and an $m c r-1$-bearing IncI2 plasmid in a single Escherichia coli ST167 clinical isolate. Infect Drug Resist. 2019;12:511-519. doi:10.2147/IDR.S192998

50. Huang J, Ma S, Yu Q, et al. Whole genome sequence of an Escherichia coli ST410 isolate co-harbouring bla $a_{\mathrm{NDM}-5}$, bla $a_{\mathrm{OXA}-1}$, $b l a_{\mathrm{CTX}-\mathrm{M}-15}$, bla $a_{\mathrm{CMY}-2}$, aac (3)-IIa and aac (6')-Ib-cr genes isolated from a patient with bloodstream infection in China. $J$ Glob Antimicrob Resist. 2019;19:354-355. doi:10.1016/j.jgar.2019.10.027

51. Naha S, Sands K, Mukherjee S, Saha B, Dutta S, Basu S. OXA-181Like carbapenemases in Klebsiella pneumoniae ST14, ST15, ST23, ST48, and ST231 from septicemic neonates: coexistence with NDM-5, resistome, transmissibility, and genome diversity. mSphere. 2021;6(1). doi:10.1128/mSphere.01156-20

52. Mei YF, Liu PP, Wan LG, et al. Virulence and genomic feature of a virulent Klebsiella pneumoniae sequence type 14 strain of serotype K2 harboring bla NDM-5 $_{1}$ in China. Front Microbiol. 2017;8:335. doi:10.3389/fmicb.2017.00335

53. Hammerum AM, Hansen F, Olesen B, et al. Investigation of a possible outbreak of NDM-5-producing ST16 Klebsiella pneumoniae among patients in Denmark with no history of recent travel using whole-genome sequencing. J Glob Antimicrob Resist. 2015;3 (3):219-221. doi:10.1016/j.jgar.2015.05.003

54. Zhang R, Liu L, Zhou H, et al. Nationwide surveillance of clinical carbapenem-resistant Enterobacteriaceae (CRE) strains in China. EBioMedicine. 2017;19:98-106. doi:10.1016/j.ebiom.2017.04.032

55. Yuan Y, Li Y, Wang G, et al. bla $a_{\mathrm{NDM}-5}$ carried by a hypervirulent Klebsiella pneumoniae with sequence type 29. Antimicrob Resist Infect Control. 2019;8:140. doi:10.1186/s13756-019-0596-1

56. Zhu W, Wang X, Qin J, Liang W, Shen Z. Dissemination and stability of the bla $a_{\mathrm{NDM}-5}$-carrying IncX3-type plasmid among multiclonal Klebsiella pneumoniae isolates. mSphere. 2020;5(6). doi:10.1128/ mSphere.00917-20

57. He T, Wang Y, Sun L, Pang M, Zhang L, Wang R. Occurrence and characterization of $b l a_{\mathrm{NDM}-5}$-positive Klebsiella pneumoniae isolates from dairy cows in Jiangsu, China. J Antimicrob Chemother. 2017;72 (1):90-94. doi:10.1093/jac/dkw357
Infection and Drug Resistance

\section{Publish your work in this journal}

Infection and Drug Resistance is an international, peer-reviewed openaccess journal that focuses on the optimal treatment of infection (bacterial, fungal and viral) and the development and institution of preventive strategies to minimize the development and spread of resistance. The journal is specifically concerned with the epidemiology of antibiotic resistance and the mechanisms of resistance development and diffusion in both hospitals and the community. The manuscript management system is completely online and includes a very quick and fair peerreview system, which is all easy to use. Visit http://www.dovepress.com/ testimonials.php to read real quotes from published authors. 